\section{(6) OPEN ACCESS}

\title{
Initial combination therapy with ambrisentan and tadalafil in connective tissue disease-associated pulmonary arterial hypertension (CTD-PAH): subgroup analysis from the AMBITION trial
}

\author{
John Gerry Coghlan, ${ }^{1}$ Nazzareno Galiè, ${ }^{2}$ Joan Albert Barberà, ${ }^{3,4}$ Adaani E Frost, ${ }^{5}$ \\ Hossein-Ardeschir Ghofrani, ${ }^{6}$ Marius M Hoeper, ${ }^{7}$ Masataka Kuwana, ${ }^{8}$ \\ Vallerie V McLaughlin, ${ }^{9}$ Andrew J Peacock, ${ }^{10}$ Gérald Simonneau, $^{11,12,13}$ \\ Jean-Luc Vachiéry, ${ }^{14}$ Christiana Blair, ${ }^{15}$ Hunter Gillies, ${ }^{16}$ Karen L Miller, ${ }^{15}$ \\ Julia H N Harris, ${ }^{17}$ Jonathan Langley, ${ }^{17}$ Lewis J Rubin, ${ }^{18}$ for the AMBITION \\ investigators
}

\begin{abstract}
Handling editor Tore K Kvien
- Additional material is published online only. To view please visit the journal online (http://dx.doi.org/10.1136/ annrheumdis-2016-210236)

For numbered affiliations see end of article.
\end{abstract}

\section{Correspondence to} Dr John Gerry Coghlan, Cardiology Department, Royal Free Hospital, Pond Street, Hampstead, London NW3 2QG, UK; Gerry.Coghlan@nhs.net

Received 18 July 2016 Revised 2 December 2016 Accepted 3 December 2016 Published Online First 30 December 2016

\section{ABSTRACT}

Background Patients with connective tissue diseaseassociated pulmonary arterial hypertension (CTD-PAH), in particular systemic sclerosis (SSC), had an attenuated response compared with idiopathic PAH in most trials. Thus, there is uncertainty regarding the benefit of PAH-targeted therapy in some forms of CTD-PAH.

Objective To explore the safety and efficacy of initial combination therapy with ambrisentan and tadalafil versus ambrisentan or tadalafil monotherapy in patients with CTD-PAH and SSc-PAH enrolled in the AMBITION trial.

Methods This was a post hoc analysis of patients with CTD-PAH and SSC-PAH from AMBITION, an event-driven, double-blind trial in patients with WHO functional class II/ III PAH. Treatment-naive patients were randomised 2:1:1 to once-daily initial combination therapy with ambrisentan plus tadalafil or monotherapy with ambrisentan or tadalafil, respectively. The primary endpoint was time to the first clinical failure event (first occurrence of death, hospitalisation for worsening $\mathrm{PAH}$, disease progression or unsatisfactory long-term clinical response).

Results In the primary analysis set $(\mathrm{N}=500), 187$ patients had CTD-PAH, of whom 118 had SSC-PAH. Initial combination therapy reduced the risk of clinical failure versus pooled monotherapy in each subgroup: CTD-PAH (HR 0.43 (95\% Cl 0.24 to 0.77)) and SSc-PAH (0.44 (0.22 to 0.89$)$ ). The most common AE was peripheral oedema, which was reported more frequently with initial combination therapy than monotherapy in the two PAH subgroups. The relative frequency of adverse events between those on combination therapy versus monotherapy was similar across subgroups.

Conclusions This post hoc subgroup analysis provides evidence that CTD-PAH and SSC-PAH patients benefit from initial ambrisentan and tadalafil combination therapy. Trial registration number NCT01178073, post results.

\section{INTRODUCTION}

The main aetiological subgroups in all pivotal therapeutic trials for pulmonary arterial hypertension (PAH) have been idiopathic PAH (iPAH) and connective tissue disease-associated PAH (CTD-PAH); within the CTD-PAH population, systemic sclerosis (SSc)-associated PAH (SSc-PAH) has been the leading cause. ${ }^{1-4}$ In short-term monotherapy clinical trials using prostanoids, ${ }^{156}$ endothelin receptor antagonists ${ }^{2} 7$ and phosphodiesterase type 5 (PDE5) inhibitors, ${ }^{3} 8$ the CTD population appeared to have an attenuated response to PAH-targeted therapy compared with patients with iPAH, particularly when assessed by 6 -min walking distance (6MWD). The results from longer-term event-driven trials, SERAPHIN ${ }^{9}$ and GRIPHON ${ }^{10}$ where the majority of patients received combination therapy, indicate that CTD-PAH has a similar reduction in the risk of an event as patients with $\mathrm{iPAH}$, though no breakdown for SSc-PAH is given in either trial. A recent meta-analysis comparing the response to treatment in $\mathrm{iPAH}$ and CTD-PAH concluded that the treatment of CTD-PAH was less effective than that of $\mathrm{iPAH}$ in terms of both increasing $6 \mathrm{MWD}$ and reducing the occurrence of clinical worsening. ${ }^{11}$

Observational data in patients with SSc-PAH indicate that survival also appears reduced compared with iPAH despite more modest haemodynamic dysfunction. ${ }^{12}$ By contrast, non-SSc-CTD-PAH patients exhibit similar survival curves to patients with $\mathrm{PAH}$ when receiving PAH-targeted therapy. ${ }^{13}$

The attenuated response, particularly in the short-term trials, has led to suggestions that 6MWD testing may not be an appropriate endpoint for patients with SSc-PAH. ${ }^{14}$ An increased prevalence of veno-occlusive disease, ${ }^{15}$ occult left heart involvement, ${ }^{16}$ associated lung disease ${ }^{17}$ and musculoskeletal involvement ${ }^{14}$ in patients labelled as having SSc-PAH are proposed as potential explanations for the apparent attenuated response.

The AMBITION trial, previously reported, was a phase III/IV, randomised, double-blind, event-driven trial comparing the safety and efficacy of ambrisentan and tadalafil initial combination therapy to ambrisentan or tadalafil monotherapy in treatmentnaive patients with $\mathrm{WHO} / \mathrm{New}$ York Heart 
Association functional class II or III PAH. ${ }^{18}$ AMBITION included a sizable population with CTD-PAH, thus providing an opportunity to evaluate the response to initial combination therapy versus monotherapy in a long-term, event-driven study.

We present a post hoc analysis of the CTD-PAH population, as well as the SSc subset of the CTD group. In addition, we provide data on the patients with $\mathrm{iPAH} /$ heritable $\mathrm{PAH}(\mathrm{hPAH})$ from the AMBITION study to explore if there are different responses to treatment between the $\mathrm{PAH}$ aetiologies.

\section{METHODS}

\section{Study design and oversight}

This was a post hoc subgroup analysis of the AMBITION trial that has been previously described in detail. ${ }^{18}$ Randomisation was performed centrally using an interactive voice response system. Eligible patients were stratified based on underlying aetiology of PAH (iPAH/hPAH vs non-iPAH) and WHO functional class (II vs III). Within both strata, patients were randomised 2:1:1 to initial combination therapy (ambrisentan $10 \mathrm{mg}$ plus tadalafil $40 \mathrm{mg}$ ) or to monotherapy (ambrisentan $10 \mathrm{mg}$ plus placebo or tadalafil $40 \mathrm{mg}$ plus placebo).

Monitoring and data collection were overseen by the sponsors. All reported clinical events were adjudicated by an independent clinical endpoint committee that was blinded to treatment randomisation and investigator. Statistical analyses were performed by Hartington Statistics and Data Management and were overseen by the sponsors.

\section{Patients}

Patients were aged $18-75$ years, weighed $\geq 40 \mathrm{~kg}$ and had baseline WHO functional class II or III symptoms and a diagnosis of $\mathrm{PPAH}, \mathrm{hPAH}, \mathrm{CTD}-\mathrm{PAH}$ or $\mathrm{PAH}$ associated with drugs or toxins, HIV (stable disease status) or repaired congenital heart defects. Further, all patients were required to have a total lung capacity $\geq 60 \%$ of predicted normal, a forced expiratory volume in $1 \mathrm{~s} \geq 55 \%$ of predicted normal, and at the start of enrolment, a mean pulmonary artery pressure $\geq 25 \mathrm{~mm} \mathrm{Hg}$, a pulmonary capillary wedge pressure (PCWP) or left ventricular end diastolic pressure (LVEDP) $\leq 15 \mathrm{~mm} \mathrm{Hg}$ and a pulmonary vascular resistance $(\mathrm{PVR}) \geq 240$ dyne $\cdot \mathrm{s} / \mathrm{cm}^{5}$.

After 6 months of study enrolment, a blinded review of the participants' baseline demographic data revealed a relatively high prevalence of risk factors for left ventricular diastolic dysfunction. Therefore, the eligibility criteria were amended to include more rigorous haemodynamic requirements (PVR increased from $\geq 240$ to $\geq 300$ dyne $\cdot \mathrm{s} / \mathrm{cm}^{5}$ for all patients; for patients with a PVR $\geq 300$ to $<500$ dyne $\cdot s / \mathrm{cm}^{5}$, a PCWP or LVEDP $\leq 12 \mathrm{~mm} \mathrm{Hg}$; and for those with a PVR $\geq 500$ dyne.s/ $\mathrm{cm}^{5}$, a PCWP $\leq 15 \mathrm{~mm} \mathrm{Hg}$ ) and to exclude patients with $\geq 3$ of the following risk factors for left ventricular diastolic dysfunction: body mass index $\geq 30 \mathrm{~kg} / \mathrm{m}^{2}$, history of essential hypertension, diabetes mellitus and historical evidence of significant coronary artery disease. The primary analysis set (PAS) comprised the patients who fulfilled the amended inclusion criteria.

\section{Assessments}

The primary endpoint was the time from randomisation to first adjudicated clinical failure, defined as the first occurrence of death, hospitalisation for worsening PAH (any hospitalisation for worsening PAH, lung or heart/lung transplant, atrial septostomy or initiation of parenteral prostanoid therapy), disease progression (decrease of $>15 \%$ from baseline 6MWD combined with WHO functional class III or IV symptoms at two consecutive visits separated by $\geq 14$ days) or unsatisfactory long-term clinical response (any decrease from baseline 6MWD at two consecutive postbaseline clinic visits separated by $\geq 14$ days and WHO functional class III symptoms assessed at two clinic visits separated by $\geq 6$ months). The time to first occurrence of each of the individual components of the primary endpoint was also analysed.

Secondary endpoints included change from baseline at week 24 in N-terminal pro-B-type natriuretic peptide (NT-proBNP) level, satisfactory clinical response to therapy (10\% improvement in 6MWD compared with baseline, with improvement to or maintenance of WHO functional class I or II symptoms, and no events of clinical worsening before or at the week 24 visit), 6MWD, Borg dyspnoea index and WHO functional class. Safety endpoints included adverse events (AEs) and laboratory assessments.

Efficacy and safety assessments were performed at screening and randomisation visits; at weeks 4, 8, 16, 24 and every 12 weeks thereafter; at the final assessment visit; and at the end-of-study visit. Laboratory assessments were performed monthly.

\section{Statistical analyses}

The PAS included all randomised patients who received study drug and met the amended entry criteria. Analyses are presented by diagnosis of PAH; because these subgroup analyses are post hoc, $\mathrm{p}$ values are not presented. The Kaplan-Meier product limit method was used to generate survival curves for time from randomisation to first adjudicated clinical failure. Cox proportional-hazards regression models were used to calculate the HR and 95\% CI. NT-proBNP levels are presented as the geometric mean and geometric mean ratio and were analysed using mixed models repeated measures. The percentage of patients with a satisfactory clinical response was analysed as a binary endpoint using logistic regression, with no imputation for missing values. 6MWD was analysed using the stratified Wilcoxon rank-sum test; missing values were imputed using last observation carried forward or worst rank scores for missing data following death or adjudicated hospitalisation for PAH.

Among patients recorded as having 'other' forms of CTD-PAH, 12 of 26 were further described as having CREST syndrome (calcinosis cutis, Raynaud's phenomenon, oesophageal dysfunction, sclerodactyly and telangiectasias), or limited or diffuse cutaneous SSc. To explore the possibility that misclassification of these patients led to a bias in favour of a treatment effect in the SSc-PAH population, a sensitivity analysis on the primary endpoint was performed where these patients were assigned to the SSc-PAH population.

\section{RESULTS}

A total of 187 of 500 patients in the PAS had CTD-PAH, and slightly more than half $(n=103)$ of patients with CTD-PAH, were randomised to initial combination therapy and 84 to pooled monotherapy. SSc-PAH comprised 63\% (118/187) of the CTD-PAH population. Figure 1 shows the distribution of this population among the treatment arms, and table 1 the full breakdown of CTD sub-aetiologies.

Among patients with CTD-PAH, those in the SSc-PAH subset tended to be older than the non-SSc-PAH population (mean 61.5 vs 52.6 years). Haemodynamic severity of disease at diagnosis was similar between the SSc-PAH subset and the non-SSc-PAH population. Baseline characteristics in patients randomised to combination therapy compared with pooled monotherapy were generally well balanced, though some differences were seen in the SSc-PAH group, where those randomised 
Figure 1 Flow chart of population distribution among the treatment arms. The modified intention-to-treat (mITT) population includes patients who were randomised and received study drug. The ex-primary analysis set (PAS) population includes patients who were randomised and received study drug but did not meet the amended entry criteria. The PAS population includes patients who were randomised, received study drug, and met the amended entry criteria. The last row of this figure is a post hoc summary. CTD, connective tissue disease; FAV, final assessment visit; $\mathrm{PAH}$, pulmonary arterial hypertension; SSc, systemic sclerosis.

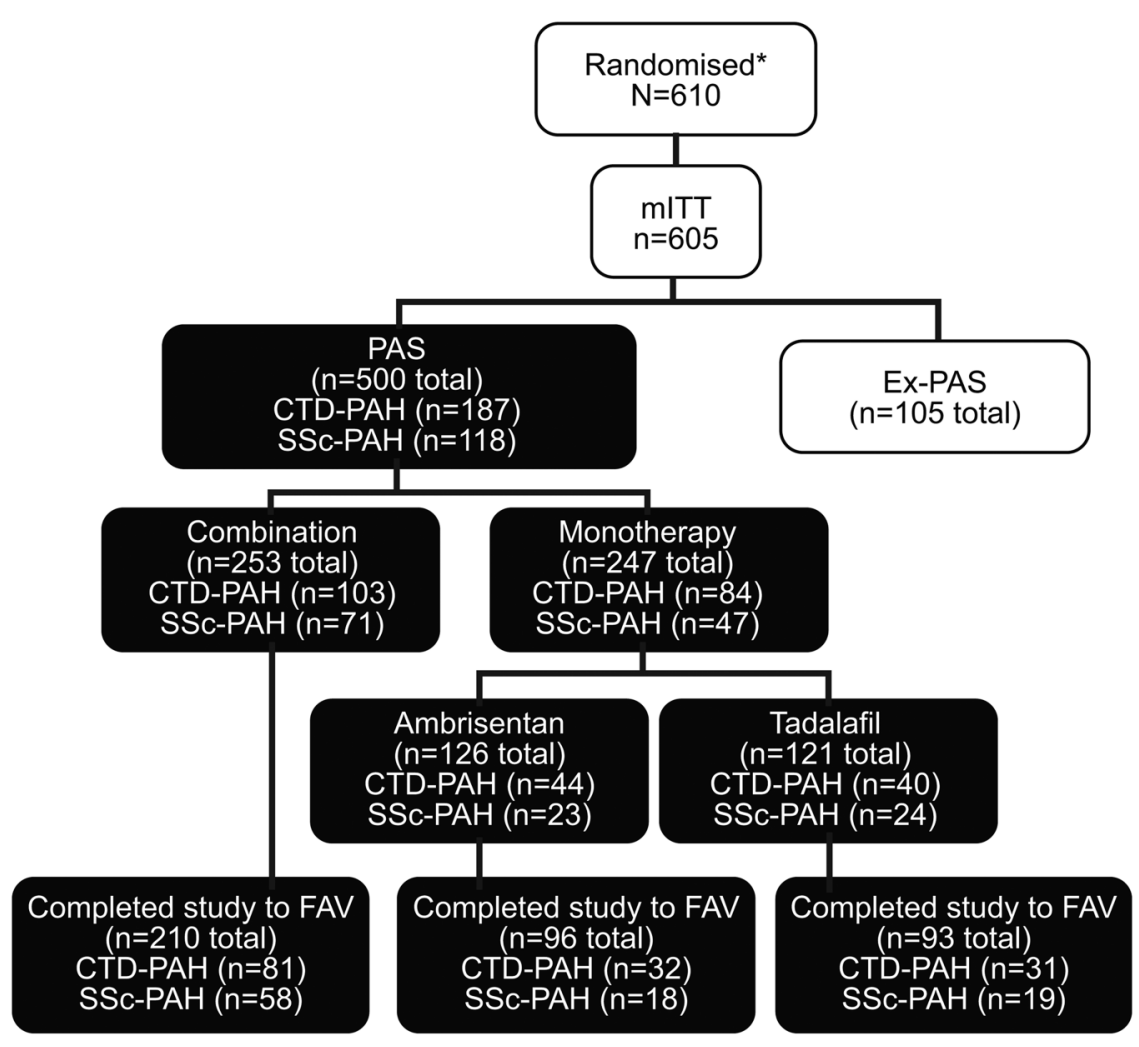

*Five randomised patients did not receive study drug. to combination therapy had a lower 6MWD, higher NT-proBNP level and a lower use of immunosuppressants. No additional obvious differences were observed between the combination therapy arm and each of the individual monotherapy arms. Table 2 summarises baseline characteristics and haemodynamics for the CTD-PAH and SSc-PAH populations, and online supplementary table $S 1$ provides the same data for SSc-PAH and non-SSc-CTD patients.

\section{Primary endpoint}

Among patients randomised to initial combination therapy, the risk of experiencing a first clinical failure event in the population with CTD-PAH was reduced compared with those on initial monotherapy with HRs of 0.43 (95\% CI 0.24 to 0.77 ) versus pooled monotherapy (figure $2 \mathrm{~A}$ ), 0.51 (95\% CI 0.25 to 1.01) versus ambrisentan monotherapy and 0.40 (95\% CI 0.20 to 0.77$)$ versus tadalafil monotherapy. The HRs correspond to risk reductions of $57 \%, 49 \%$ and $60 \%$, respectively. The magnitude of benefit of combination therapy over monotherapy was similar in the SSc-PAH population with HRs of 0.44 (95\% CI 0.22 to 0.89 ) versus pooled monotherapy (figure $2 \mathrm{~B}$ ), 0.52 (95\% CI 0.21 to 1.26 ) versus ambrisentan monotherapy and 0.44 (95\% CI 0.20 to 0.97 ) versus tadalafil monotherapy. The HRs correspond to risk reductions of $56 \%, 48 \%$ and $56 \%$, respectively.

Overall in CTD-PAH, 19\% (20/103) of combination therapy patients experienced a primary endpoint event and 36\% (30/84) of monotherapy patients experienced a primary endpoint event; in SSc-PAH, it was $21 \%(15 / 71)$ of combination therapy and $40 \%(19 / 47)$ of monotherapy patients.

The results for the time to first occurrence of the four individual components of the primary endpoint by $\mathrm{PAH}$ type, along 
Table 2 Baseline characteristics and haemodynamics among patients with CTD-PAH and SSc-PAH

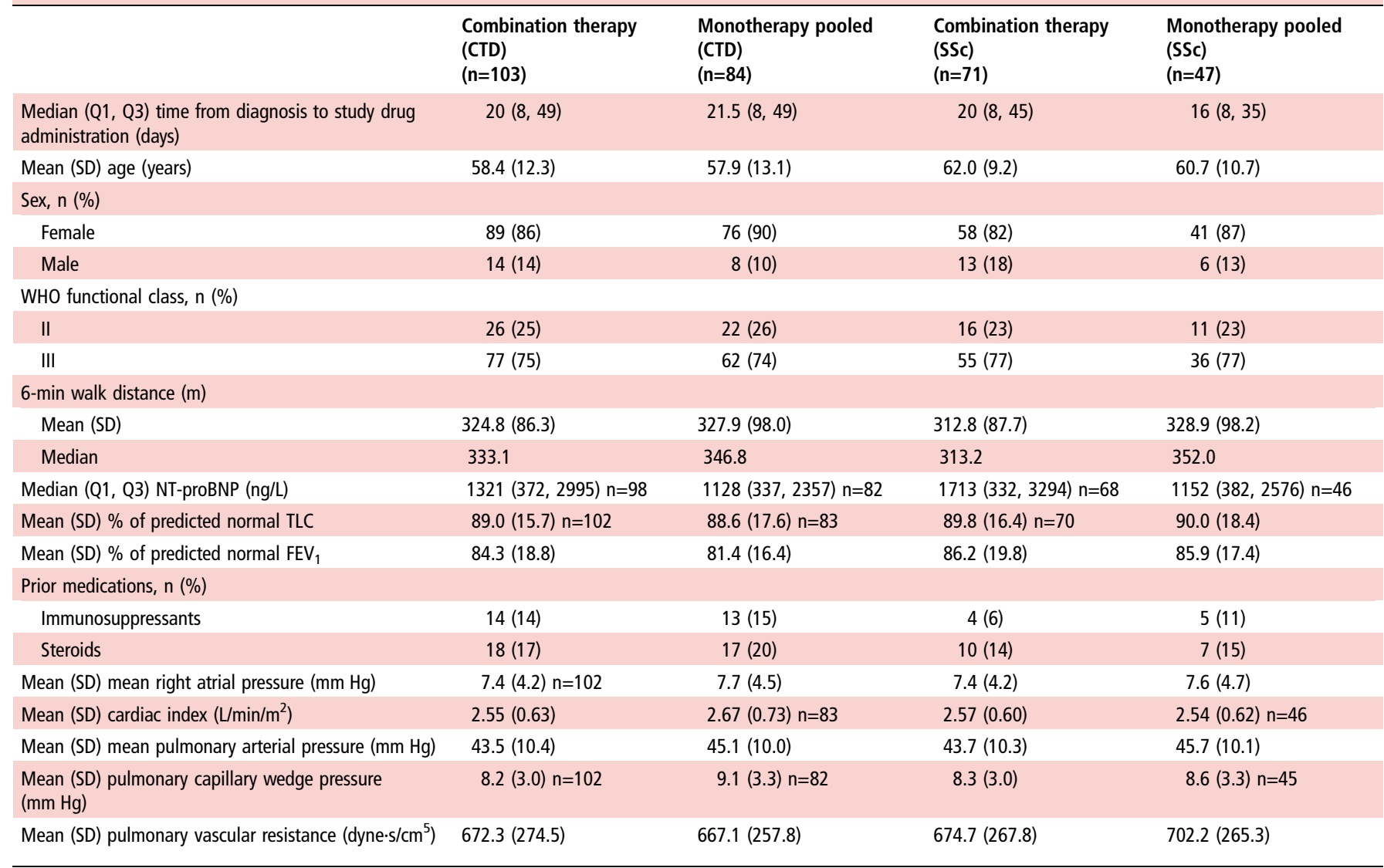

\section{Post hoc summary.}

CTD, connective tissue disease; FEV 1 , forced expiratory volume in $1 \mathrm{~s}$; NT-proBNP, N-terminal pro-B-type natriuretic peptide; PAH, pulmonary arterial hypertension; Q1, quartile 1; Q3, quartile 3; SSc, systemic sclerosis; TLC, total lung capacity.

with an analysis of time to clinical worsening (where the unsatisfactory long-term clinical response component is removed from the composite endpoint), are presented in figure 3. Time to first hospitalisation for worsening PAH showed the greatest difference between combination therapy and monotherapy for both the overall CTD-PAH population (risk reduction: 71\%; HR 0.29 (95\% CI 0.12 to 0.67$)$ ) and the SSc-PAH population (risk reduction: 64\%; HR 0.36 (95\% CI 0.13 to 1.04$)$ ).

\section{Sensitivity analysis}

In the sensitivity analysis, patients classified as other-CTD-PAH on the case report form but further described as having SSc-PAH were included, increasing the number of patients in the SSc-PAH subset from 118 to 130 . Of these, 75 patients received initial combination therapy and 55 were randomised to monotherapy. The HR for the primary endpoint sensitivity analysis was 0.47 (95\% CI 0.24 to 0.92 ), corresponding to a $53 \%$ risk reduction and indicating that any misclassification had little impact on the reported outcomes.

\section{Secondary endpoints}

At week 24, mean reduction in NT-proBNP from baseline was consistent between diagnosis subgroups and greater in patients receiving combination therapy versus pooled monotherapy: overall CTD-PAH (-60.4\% vs $-43.1 \%)$ and SSc-PAH $(-62.8 \%$ vs $-38.4 \%)$. The corresponding geometric mean ratios expressed as \% differences were $-30.4 \%$ (95\% CI -49.0 to $-5.2)$ and $-39.6 \%$ (95\% CI -59.3 to -10.2$)$ for the two populations, respectively. Rates of satisfactory clinical response at week 24 were similar with combination therapy and pooled monotherapy in the overall CTD-PAH (35\% combination, 29\% monotherapy; OR 1.321 (95\% CI 0.680 to 2.565$)$ ) and SSc-PAH populations (31\% combination, 29\% monotherapy; OR 1.111 (95\% CI 0.474 to 2.604$)$ ). A greater increase in median 6MWD from baseline was seen at week 24 in patients receiving initial combination therapy versus pooled monotherapy in the overall CTD-PAH $(+42.0$ vs $+24.3 \mathrm{~m})$ and SSc-PAH $(+40.9$ vs $+12.2 \mathrm{~m})$ populations. The corresponding median differences (combination-monotherapy) were $+19.7 \mathrm{~m} \quad(95 \%$ $\mathrm{CI}+1.0$ to +38.4$)$ and $+30.3 \mathrm{~m}(95 \% \mathrm{CI}+4.0$ to +56.5$)$ for the two populations, respectively. In a post hoc analysis, the proportion of patients with $\mathrm{a} \geq 15 \%$ decrease in $6 \mathrm{MWD}$ at any time post baseline was greater with pooled monotherapy than with combination therapy across all subgroups (CTD$\mathrm{PAH}, \mathrm{SSc}-\mathrm{PAH}$ and non-SSc-PAH) (see online supplementary table S2).

\section{Safety}

Post hoc summaries indicate that patients with CTD-PAH were treated for a mean (SD) of 584 (356) days in the combination therapy arm and 499 (329) days in the pooled monotherapy arm, and patients with SSc-PAH were treated for 566 (363) days and 504 (330) days, respectively. No new safety signal was identified in the overall CTD-PAH or SSc-PAH populations. The most common AEs $(\geq 25 \%)$ in the combination therapy group for these two populations were peripheral oedema $(47 \%$ 
Figure 2 Kaplan-Meier curves for the time from randomisation to first adjudicated clinical failure in the (A) connective tissue disease-associated pulmonary arterial hypertension population and (B) systemic sclerosis-pulmonary arterial hypertension population. Post hoc figures. The HR is for combination versus pooled monotherapy.
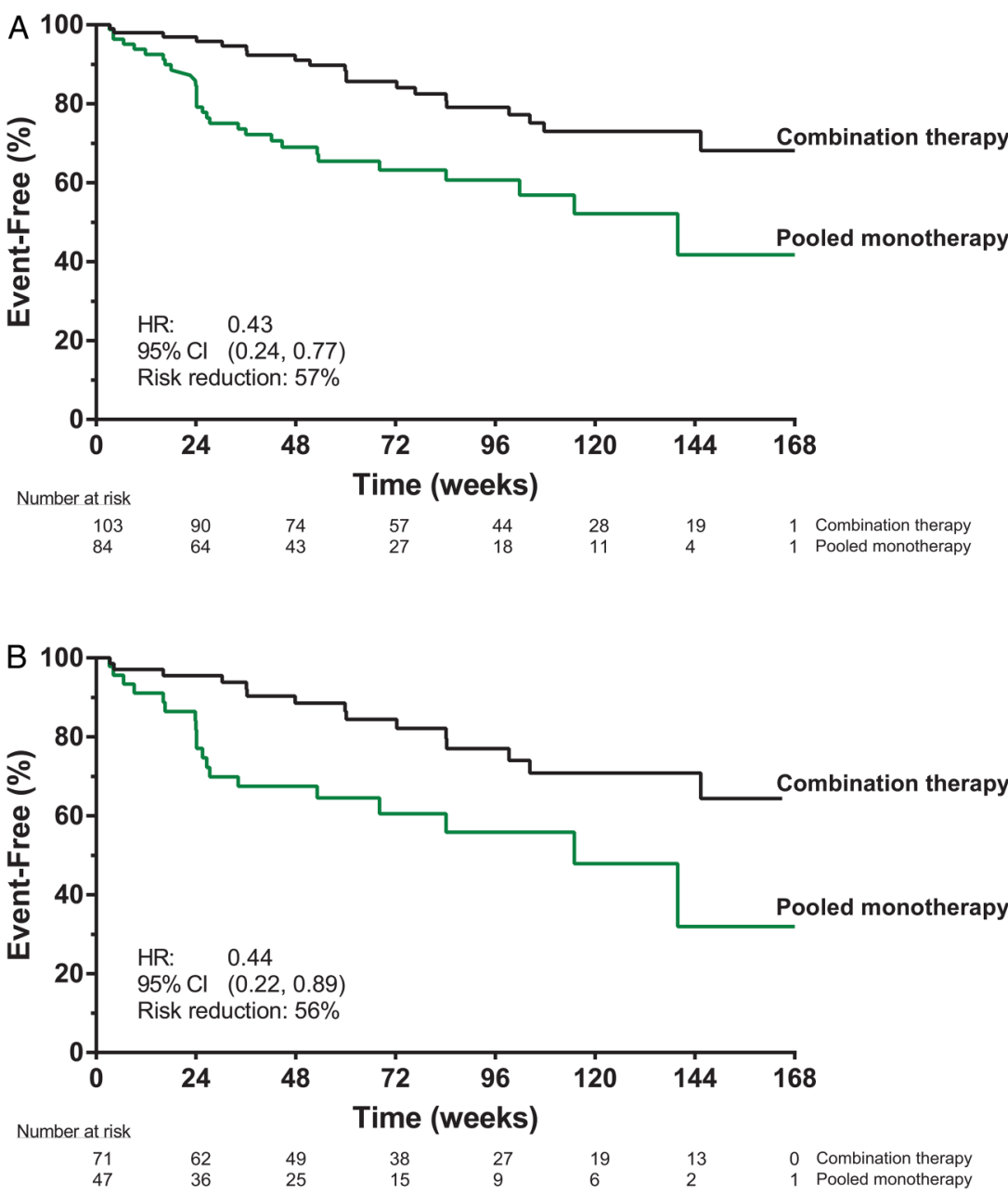

combination, 34\% ambrisentan and 33\% tadalafil in CTD-PAH; $45 \%, 26 \%$ and $33 \%$, respectively, in SSc-PAH), headache $(33 \%$ combination, 32\% ambrisentan and 38\% tadalafil in CTD-PAH; $28 \%, 26 \%$ and 33\%, respectively, in SSc-PAH) and diarrhoea (29\% combination, 32\% ambrisentan and 25\% tadalafil in CTD-PAH; 28\%, 17\% and 29\%, respectively, in SSc-PAH). Anaemia occurred at a similar incidence in the combination therapy and tadalafil monotherapy groups, but was not reported in the ambrisentan monotherapy group (19\% combination, $0 \%$ ambrisentan and 15\% tadalafil in CTD-PAH; 21\%, $0 \%$ and $17 \%$, respectively, in SSc-PAH). Overall rates of serious adverse events (SAEs) and AEs leading to permanent discontinuation of study drug were similar in the overall CTD-PAH and SSc-PAH populations, with no trend towards an excess of such events with initial combination therapy compared with monotherapy (table 3 ). In patients with CTD-PAH, SAE rates were $44 \%$ with combination therapy, 34\% with ambrisentan monotherapy and $50 \%$ with tadalafil monotherapy. SAE rates were similar in patients with SSc-PAH (44\%, 39\% and 58\%, respectively).

\section{Comparison with the iPAH/hPAH subgroup}

Baseline characteristics indicated some similarities and differences between patients in the $\mathrm{iPAH} / \mathrm{hPAH}$ subgroup and those in the CTD-PAH and SSc-PAH subgroups. Patients with $\mathrm{iPAH} / \mathrm{hPAH}$ had similar functional status, but they were, on average, younger and had a longer 6MWD, lower NT-proBNP level and worse haemodynamics (see online supplementary table S3). Results for the primary endpoint (see online supplementary figure S1) and individual components of the primary endpoint (see online supplementary figure S2) were similar in the iPAH/ hPAH population compared with the CTD-PAH and SSc-PAH populations. For the primary endpoint in the $\mathrm{iPAH} / \mathrm{hPAH}$ population, initial combination therapy reduced the risk of experiencing a first clinical failure event compared with initial monotherapy (risk reduction: 49\%; HR 0.51 (95\% CI 0.31 to $0.83))$. Overall, in patients with iPAH/hPAH 19\% (25/134) of the combination group and $32 \%(46 / 145)$ of the monotherapy group experienced a primary endpoint event. Among the secondary endpoints in the patients with $\mathrm{iPAH} / \mathrm{hPAH}$, mean change in NT-proBNP from baseline at week 24 was $-71.2 \%$ with combination therapy and $-50.0 \%$ with pooled monotherapy; geometric mean ratio expressed as percentage difference was $-42.5 \%$ (95\% CI -54.6 to -27.0$)$. Compared with pooled monotherapy, combination therapy was associated with a greater proportion of patients with a satisfactory clinical response at week 24 (40\% vs 28\%, OR 1.736 (95\% CI 1.035 to 2.911)) and a greater increase in median 6MWD from baseline at week $24(+52.5$ vs $+26.6 \mathrm{~m})$. The corresponding median difference was $+26.6 \mathrm{~m}(95 \% \mathrm{CI}+11.6$ to +41.5$)$.

Among patients with $\mathrm{iPAH} / \mathrm{hPAH}$, AEs occurring in $\geq 25 \%$ on initial combination therapy were peripheral oedema $(48 \%$ combination vs $31 \%$ ambrisentan and $27 \%$ tadalafil) and headache (45\% vs $32 \%$ and $31 \%$, respectively). Other AEs as well as rates of SAEs and AEs leading to permanent discontinuation of study drug were more evenly distributed among treatment groups. Rates of SAEs and AEs leading to permanent discontinuation of 


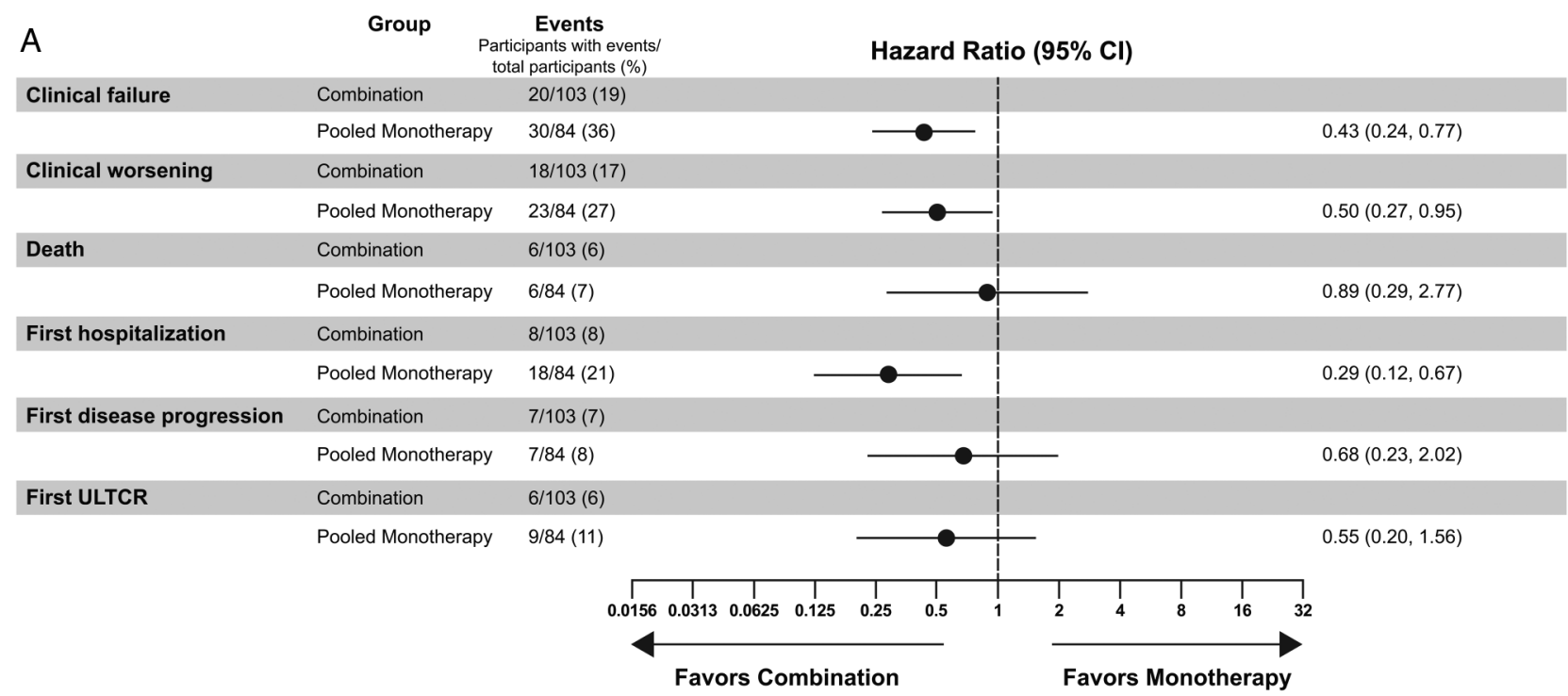

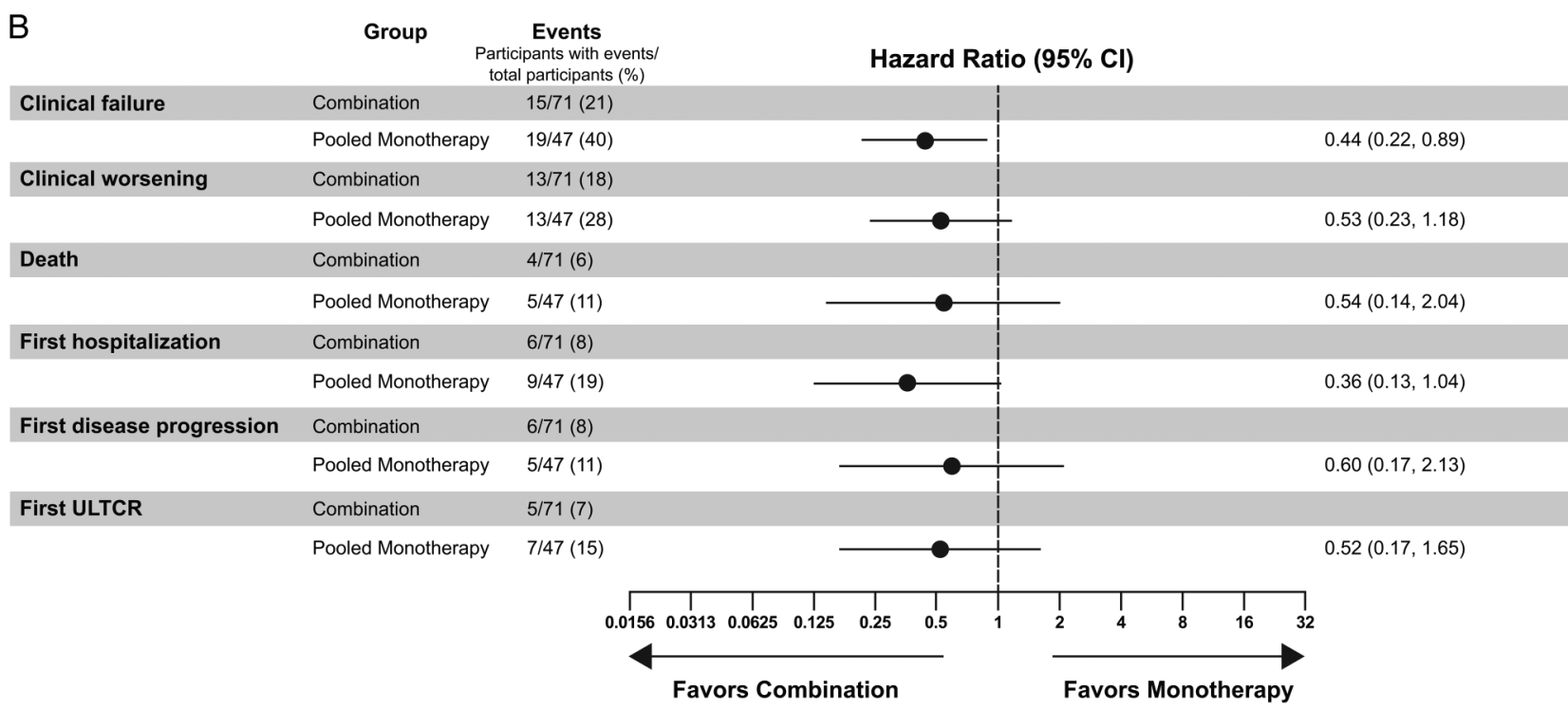

Figure 3 Forest plot of time to first occurrence of clinical failure, clinical worsening, death, hospitalisation, disease progression and unsatisfactory long-term clinical response (ULTCR) in the (A) connective tissue disease-associated pulmonary arterial hypertension population and (B) systemic sclerosis-pulmonary arterial hypertension population. Post hoc figures. The HR is for combination versus pooled monotherapy.

study drug were lower in the iPAH/hPAH population (see online supplementary table S4) compared with the CTD-PAH and SSc-PAH populations. In patients with $\mathrm{iPAH} / \mathrm{hPAH}$, rates of SAEs were $33 \%$ with combination therapy, 37\% with ambrisentan monotherapy and $39 \%$ with tadalafil monotherapy, and rates of AEs leading to permanent discontinuation of study drug were $11 \%, 8 \%$ and $11 \%$, respectively.

\section{DISCUSSION}

The AMBITION trial included a sizable number of patients with CTD-PAH, and within this SSc-PAH, and thus provides an opportunity to explore whether this population responds to initial combination therapy with ambrisentan and tadalafil compared with monotherapy of either agent. Initial combination therapy resulted in a reduction in the risk of clinical failure versus pooled monotherapy of $57 \%$ in patients with CTD-PAH and $56 \%$ in the subset of patients with SSc-PAH. While inferential statistics are not presented, the 95\% CIs around the HR for both CTD-PAH and SSc-PAH were well within the bounds of
$0-1$. This result is further supported by the secondary endpoints, where we see a greater improvement in plasma NT-proBNP levels and 6MWD on combination therapy compared with monotherapy. The benefit of combination therapy over monotherapy is of similar magnitude to that observed in the whole trial population.

No new safety signals were identified in the CTD-PAH subset. However, some AEs appeared to be more frequent in CTD-PAH than in the iPAH/hPAH population (eg, diarrhoea and anaemia), possibly due to coexisting connective tissue disease.

This subgroup analysis offers a number of insights that are informative to the management of patients with CTD-PAH. As previously reported, patients with CTD-PAH tend to have more modest haemodynamic dysfunction than patients with iPAH/ hPAH, though do not appear to have a better outcome. It has been reported that the CTD-PAH population, particularly $\mathrm{SSc}-\mathrm{PAH}$, has a relatively poor prognosis, and their response to $\mathrm{PAH}$-targeted monotherapies in short-term trials (eg, reduction of clinical worsening events, improvements in exercise ability) 
Table 3 Summary of adverse events among patients with CTD-PAH and SSC-PAH

\begin{tabular}{|c|c|c|c|c|c|c|}
\hline \multirow[b]{2}{*}{ n (\%) } & \multicolumn{3}{|l|}{ CTD-PAH } & \multicolumn{3}{|c|}{ SSC-PAH } \\
\hline & $\begin{array}{l}\text { COMB } \\
(n=103)\end{array}$ & $\begin{array}{l}\text { AMB mono } \\
(n=44)\end{array}$ & $\begin{array}{l}\text { TAD mono } \\
(n=40)\end{array}$ & $\begin{array}{l}\text { COMB } \\
(n=71)\end{array}$ & $\begin{array}{l}\text { AMB mono } \\
(n=23)\end{array}$ & $\begin{array}{l}\text { TAD mono } \\
(n=24)\end{array}$ \\
\hline Any $A E^{*}$ & $102(99)$ & $42(95)$ & $39(98)$ & 70 (99) & $22(96)$ & $23(96)$ \\
\hline Oedema peripheral & $48(47)$ & $15(34)$ & $13(33)$ & $32(45)$ & $6(26)$ & $8(33)$ \\
\hline Headache & $34(33)$ & $14(32)$ & $15(38)$ & $20(28)$ & $6(26)$ & $8(33)$ \\
\hline Diarrhoea & $30(29)$ & $14(32)$ & $10(25)$ & $20(28)$ & $4(17)$ & $7(29)$ \\
\hline Pulmonary hypertension $\ddagger$ & $7(7)$ & $3(7)$ & $6(15)$ & $4(6)$ & 0 & $5(21)$ \\
\hline Pneumonia & $6(6)$ & $3(7)$ & $2(5)$ & $3(4)$ & $3(13)$ & $1(4)$ \\
\hline Dyspnoea & $4(4)$ & $2(5)$ & $2(5)$ & $4(6)$ & $1(4)$ & $2(8)$ \\
\hline Anaemia & $3(3)$ & 0 & $3(8)$ & $3(4)$ & 0 & $1(4)$ \\
\hline Syncope & $2(2)$ & $1(2)$ & 1 (3) & 0 & 0 & 0 \\
\hline Diarrhoea & $2(2)$ & 0 & 0 & $1(1)$ & 0 & 0 \\
\hline Nausea & $2(2)$ & 0 & 0 & $2(3)$ & 0 & 0 \\
\hline Headache & $2(2)$ & 0 & 0 & $1(1)$ & 0 & 0 \\
\hline Dyspnoea & $1(<1)$ & 0 & 0 & $1(1)$ & 0 & 0 \\
\hline Pulmonary oedema & 0 & $1(2)$ & 0 & 0 & $1(4)$ & 0 \\
\hline
\end{tabular}

Post hoc summary.

*AEs occurring in $\geq 25 \%$ of patients on combination therapy in either the CTD-PAH or SSC-PAH populations.

TSAEs occurring in $\geq 4 \%$ of patients on combination therapy in either the CTD-PAH or SSc-PAH populations; only the iPAH/hPAH population met this criterion for syncope (after rounding) (see online supplementary table $\mathrm{S4}$ ), but rates are shown in this table to allow for comparison.

fIn each case, the investigator reported the events using additional text not captured in the preferred term, describing this as worsening of pulmonary hypertension. However, an AE report of worsening pulmonary hypertension does not necessarily become a primary endpoint event, which has specific criteria.

§AEs leading to permanent study drug discontinuation in $\geq 2$ patients on combination therapy in either the CTD-PAH or SSc-PAH populations; only the iPAH/hPAH population met this criterion for dyspnoea and pulmonary oedema (see online supplementary table S4), but rates are shown in this table to allow for comparison.

$A E$, adverse event; $A M B$, ambrisentan; COMB, combination therapy; CTD, connective tissue disease; hPAH, heritable pulmonary arterial hypertension; iPAH, idiopathic pulmonary arterial hypertension; Mono, monotherapy; PAH, pulmonary arterial hypertension; SAE, serious adverse event; SSc, systemic sclerosis; TAD, tadalafil.

has, with the exception of the initial epoprostenol trial, ${ }^{19}$ not been as robust as in the iPAH/hPAH population.

The present findings in the monotherapy arm support this, with $32 \%$ of patients with $\mathrm{iPAH} / \mathrm{hPAH}, 36 \%$ of patients with CTD-PAH and $40 \%$ of patients with SSc-PAH experiencing a clinical failure event. However, the combination therapy arm of $\mathrm{iPAH} / \mathrm{hPAH}, \mathrm{CTD}-\mathrm{PAH}$ and SSc-PAH had an overall incidence of a clinical failure event of $19 \%, 19 \%$ and $21 \%$, respectively. This corresponds to a risk reduction for combination therapy of $49 \%$ in $\mathrm{iPAH} / \mathrm{hPAH}, 57 \%$ in CTD-PAH and $56 \%$ in SSc-PAH compared with pooled monotherapy, suggesting that initial oral combination therapy in the CTD-PAH population may result in similar outcomes to that observed in the iPAH/hPAH patient group, at least over the duration of this study.

This is supported by the results of the $6 \mathrm{MWD}$, particularly for SSc-PAH, where monotherapy provided a limited improvement from baseline over 24 weeks $(12.2 \mathrm{~m})$, which is in line with the previous short-term monotherapy trials. However, initial combination therapy resulted in a $40.9 \mathrm{~m}$ improvement from baseline, indicating perhaps that the 6MWD may not be an inappropriate endpoint in patients with SSc-PAH and may be a useful indicator of response to treatment.

Additionally, a $15 \%$ worsening of $6 \mathrm{MWD}$ has been associated with a significantly worse prognosis in the REVEAL study. ${ }^{20}$ In AMBITION, the number of subjects with a $\geq 15 \%$ worsening at any time was $31 / 131$ (24\%) with combination therapy compared with 46/144 (32\%) with monotherapy in the iPAH/hPAH group and similarly was $21 / 67$ (31\%) on combination therapy compared with 20/45 (44\%) on monotherapy in the SSc-PAH group over the course of this long-term study (see online supplementary table S2).

These results support the notion that CTD-PAH, particularly SSc-PAH, may need a more aggressive treatment regimen to see similar benefits to patients with iPAH.

In AMBITION, a protocol amendment was made to allow for a more rigorous approach of excluding postcapillary pulmonary venous hypertension from the PAS population, ${ }^{18}$ which may have provided a possible explanation for the results. However, an analysis of the modified intent-to-treat population (all randomised patients who received study drug), which includes a further 29 patients with CTD-PAH (19 of whom were SSc-PAH), shows similar results, with risk reductions of $52 \%$ for CTD-PAH (HR 0.48 ; 95\% CI 0.29 to 0.82 ) and $54 \%$ for SSc-PAH (HR 0.46; 95\% CI 0.24 to 0.90 ), indicating that excluding patients who failed to meet the revised inclusion criteria had limited impact on the outcome. Other eligibility criteria, including pulmonary function parameters, remained unchanged and in line with other PAH trials. The purpose of the pulmonary function test (PFT) parameters is to exclude those patients whose $\mathrm{PH}$ was likely driven by lung disease. However, such restrictions would not remove the possibility of including patients with a modest degree of pulmonary fibrosis or exclude the possibility of pulmonary veno-occlusive disease in the CTD-PAH population.

Our data are supported by a recent study published by Hassoun and colleagues. ${ }^{21}$ In this open-label study of 24 
treatment-naive patients diagnosed with SSc-PAH, the initiation of treatment with ambrisentan $(10 \mathrm{mg})$ and tadalafil $(40 \mathrm{mg})$ daily for 36 weeks resulted in a statistically significant improvement from baseline in the co-primary endpoint of reduction in right ventricular mass and PVR. There were also significant improvements in the secondary endpoints, including an estimate of pulmonary artery compliance (stroke volume/pulmonary pulse pressure ratio), echo-measured tricuspid annular plane systolic excursion, 6MWD, functional class and serum NT-proBNP. The most common AEs included fluid accumulation (29\%), headache (29\%) and nasal congestion (16\%); SAEs were rare and limited, and included one case of fluid overload requiring hospitalisation.

Our study has several limitations. These were post hoc analyses of the subgroups with CTD-PAH and SSc-PAH. Given the small number of non-SSc-CTD-PAH patients, no reliable comparisons of treatment response between the CTD sub-aetiologies are possible. Twelve patients were classified as 'other' CTD-PAH when subsequent descriptors suggested that these patients may have had SSc-PAH; however, a sensitivity analysis showed that this did not affect the overall findings.

\section{CONCLUSIONS}

This post hoc analysis of patients with CTD-PAH in AMBITION suggests that this subpopulation did at least as well on initial combination therapy compared with patients with $\mathrm{iPAH} / \mathrm{hPAH}$, both in terms of clinical failure risk reduction and improvement in exercise ability. There were no new safety signals observed in the CTD-PAH cohort compared with the full study results, though rates of SAEs and AEs leading to permanent discontinuation of study drug were higher in the CTD-PAH group than the $\mathrm{iPAH} / \mathrm{HPAH}$ group. In the CTD-PAH population, an aggressive approach to treatment with initial combination therapy may improve outcomes and exercise capacity as opposed to treatment with monotherapy.

\author{
Author affiliations \\ ${ }^{1}$ Cardiology Department, Royal Free Hospital, London, UK \\ ${ }^{2}$ Department of Experimental, Diagnostic and Specialty Medicine-DIMES, University \\ of Bologna, Bologna, Italy \\ ${ }^{3}$ Department of Respiratory Medicine, Hospital Clínic-Institut d'Investigacions \\ Biomèdiques August Pi i Sunyer, University of Barcelona, Barcelona, Spain \\ ${ }^{4}$ Biomedical Research Networking Center on Respiratory Diseases, Madrid, Spain \\ ${ }^{5}$ Houston Methodist Lung Center, Houston, Texas, USA \\ ${ }^{6}$ Universities of Giessen and Marburg Lung Center (UGMLC), Giessen, Germany \\ ${ }^{7}$ Hannover Medical School and German Center of Lung Research (DZL) Hannover, \\ Hannover, Germany \\ ${ }^{8}$ Department of Allergy and Rheumatology, Nippon Medical School Graduate School \\ of Medicine, Tokyo, Japan \\ ${ }^{9}$ Univeristy of Michigan Health System, Ann Arbor, Michigan, USA \\ ${ }^{10}$ Regional Heart and Lung Centre, Glasgow, UK \\ ${ }^{11}$ Faculté de Médecine, Université Paris-Sud, Le Kremlin Bicêtre, France \\ ${ }^{12}$ Département Hospitalo-Universitaire (DHU) Thorax Innovation (TORINO), Service de \\ Pneumologie, AP-HP, Centre de Référence de I'Hypertension Pulmonaire Sévère, \\ Hôpital de Bicêtre, Le Kremlin Bicêtre, France \\ ${ }^{13}$ Laboratoire d'Excellence (LabEx) en Recherche sur le Médicament et I'Innovation \\ Thérapeutique (LERMIT), UMR_S 999, INSERM, Centre Chirurgical Marie \\ Lannelongue, Le Plessis Robinson, France \\ ${ }^{14}$ Universitaires de Bruxelles-Hôpital Erasme, Brussels, Belgium \\ ${ }^{15}$ Gilead Sciences, Inc., Foster City, California, USA \\ ${ }^{16}$ Former employee of Gilead Sciences, Inc., Foster City, California, USA \\ ${ }^{17}$ GlaxoSmithKline, Uxbridge, UK \\ ${ }^{18}$ University of California at San Diego, La Jolla, California, USA
}

Acknowledgements Editorial support was provided by Michael S. McNamara, MS, and Jessica Holzhauer, DVM, of C4 MedSolutions, LLC (Yardley, PA), a CHC Group company, with funding from Gilead Sciences, Inc and GlaxoSmithKline.

Contributors JGC contributed to the analysis and drafted the manuscript. All other authors critically revised the manuscript and approved the final version. All authors contributed to the planning and/or the conduct of the study.
Funding This study was supported by Gilead Sciences and GlaxoSmithKline.

Competing interests JGC: grants and personal fees from Actelion and GlaxoSmithKline; personal fees from Bayer, Endotronix, Pfizer and United Therapeutics. NG: grants and personal fees from Actelion, Bayer, GlaxoSmithKline and Pfizer. JAB: personal fees from Actelion, Almirall, Bayer, GlaxoSmithKline and Pfizer; grants from Actelion, Bayer, GlaxoSmithKline and Pfizer. AEF: funds for the conduct of the study from Baylor College of Medicine; honoraria and travel/lodging expense for participating on the study's Steering Committee; grants from Actelion, Bayer, Gilead and United Therapeutics; personal fees from Actelion, Bayer, Gilead, Ikaria and United Therapeutics; non-financial support from Bayer and Novartis. H-AG: grants from Actelion, Bayer, Novartis and Pfizer; board membership and consultancy for Actelion, Bayer, GlaxoSmithKline, Merck, Novartis, Pfizer and Takeda; consultancy for Lilly; payment for lectures from Actelion, Bayer,

GlaxoSmithKline, Lilly, Novartis and Pfizer. MMH: personal fees from Actelion, Bayer, Gilead, GlaxoSmithKline, Merck and Pfizer. MK: grants and personal fees from Actelion, Bayer and Pfizer; personal fees from GlaxoSmithKline. VVM: grants and personal fees from Actelion, Bayer, Gilead, Ikaria and United Therapeutics; grants from Novartis; personal fees from Steadymed and Akros. AJP: grants and personal fees from Actelion, Bayer, Gilead and GlaxoSmithKline; personal fees from United Therapeutics. GS: grants and personal fees from Actelion, Bayer, Gilead and GlaxoSmithKline. J-LV: grants from Actelion and GlaxoSmithKline; speaker fees from Actelion, Bayer, GlaxoSmithKline and Pfizer; advisory board honoraria from Actelion and Merck. CB: employee of Gilead; stock options in Gilead. HG: former employee of Gilead; patent pending in relation to the use of selective ERA's and PDE5 inhibitors. KLM: employee of Gilead; stock options in Gilead. JHNH: employee of GlaxoSmithKline; stock options in GlaxoSmithKline. JL: employee of GlaxoSmithKline. LJR: personal fees from Gilead.

\section{Patient consent Obtained.}

Ethics approval The institutional review board at each centre approved the study protocol.

Provenance and peer review Not commissioned; externally peer reviewed.

Open Access This is an Open Access article distributed in accordance with the Creative Commons Attribution Non Commercial (CC BY-NC 4.0) license, which permits others to distribute, remix, adapt, build upon this work non-commercially, and license their derivative works on different terms, provided the original work is properly cited and the use is non-commercial. See: http://creativecommons.org/ licenses/by-nc/4.0/

\section{REFERENCES}

1 Oudiz RJ, Schilz RJ, Barst RJ, et al. Treprostinil, a prostacyclin analogue, in pulmonary arterial hypertension associated with connective tissue disease. Chest 2004; 126:420-7.

2 Rubin LJ, Badesch DB, Barst RJ, et al. Bosentan therapy for pulmonary arterial hypertension. N Engl J Med 2002;346:896-903.

3 Badesch DB, Hill NS, Burgess G, et al. Sildenafil for pulmonary arterial hypertension associated with connective tissue disease. J Rheumatol 2007;34:2417-22.

4 Denton $\mathrm{CP}$, Humbert M, Rubin L, et al. Bosentan treatment for pulmonary arterial hypertension related to connective tissue disease: a subgroup analysis of the pivotal clinical trials and their open-label extensions. Ann Rheum Dis 2006:65:1336-40.

5 Barst RJ, McGoon M, McLaughlin V, et al. Beraprost therapy for pulmonary arterial hypertension. J Am Coll Cardiol 2003;41:2119-25.

6 Olschewski H, Simonneau G, Galiè N, et al. Inhaled iloprost for severe pulmonary hypertension. N Engl J Med 2002;347:322-9.

7 Galiè N, Olschewski H, Oudiz RJ, et al. Ambrisentan for the treatment of pulmonary arterial hypertension: results of the ambrisentan in pulmonary arterial hypertension, randomized, double-blind, placebo-controlled, multicenter, efficacy (ARIES) study 1 and 2. Circulation 2008;117:3010-19.

8 Galiè N, Brundage BH, Ghofrani $\mathrm{HA}$, et al. Tadalafil therapy for pulmonary arterial hypertension. Circulation 2009;119:2894-903.

9 Pulido T, Adzerikho I, Channick RN, et al. Macitentan and morbidity and mortality in pulmonary arterial hypertension. N Engl J Med 2013;369:809-18.

10 Sitbon O, Channick R, Chin KM, et al. Selexipag for the treatment of pulmonary arterial hypertension. N Engl J Med 2015;373:2522-33.

11 Rhee RL, Gabler NB, Sangani $\mathrm{S}$, et al. Comparison of treatment response in idiopathic and connective tissue disease-associated pulmonary arterial hypertension. Am J Respir Crit Care Med 2015;192:1111-17.

12 Clements PJ, Tan M, McLaughlin VV, et al. The pulmonary arterial hypertension quality enhancement research initiative: comparison of patients with idiopathic PAH to patients with systemic sclerosis-associated PAH. Ann Rheum Dis 2012;71:249-52.

13 Chung L, Farber HW, Benza R, et al. Unique predictors of mortality in patients with pulmonary arterial hypertension associated with systemic sclerosis in the REVEAL registry. Chest 2014;146:1494-504.

14 Avouac J, Wipff J, Kahan A, et al. Effects of oral treatments on exercise capacity in systemic sclerosis related pulmonary arterial hypertension: a meta-analysis of randomised controlled trials. Ann Rheum Dis 2008;67:808-14. 


\section{Clinical and epidemiological research}

15 Overbeek MJ, Vonk MC, Boonstra A, et al. Pulmonary arterial hypertension in limited cutaneous systemic sclerosis: a distinctive vasculopathy. Eur Respir J 2009;34:371-9.

16 Fox BD, Shimony A, Langleben $D$, et al. High prevalence of occult left heart disease in scleroderma-pulmonary hypertension. Eur Respir J 2013;42:1083-91.

17 Lefèvre G, Dauchet L, Hachulla E, et al. Survival and prognostic factors in systemic sclerosis-associated pulmonary hypertension: a systematic review and meta-analysis. Arthritis Rheum 2013;65:2412-23.

18 Galiè N, Barberà JA, Frost AE, et al. Initial use of ambrisentan plus tadalafil in pulmonary arterial hypertension. N Engl J Med 2015;373:834-44.
19 Badesch DB, Tapson VF, McGoon MD, et al. Continuous intravenous epoprostenol for pulmonary hypertension due to the scleroderma spectrum of disease. A randomized, controlled trial. Ann Intern Med 2000;132:425-34.

20 Farber HW, Miller DP, McGoon MD, et al. Predicting outcomes in pulmonary arteria hypertension based on the 6 minute walk distance. J Heart Lung Transplant 2015;34:362-8.

21 Hassoun PM, Zamanian RT, Damico R, et al. Ambrisentan and tadalafil up-front combination therapy in scleroderma-associated pulmonary arterial hypertension. Am J Respir Crit Care Med 2015;192:1102-10. 